\title{
Evaluation of cerebral aneurysm wall thickness in experimental aneurysms: comparison of 3T-MR imaging with direct microscopic measurements
}

\author{
Camillo Sherif ${ }^{1,2,3} \cdot$ Martin Krssak $^{4,5}$
}

Received: 10 January 2018 / Accepted: 16 January 2018 / Published online: 23 February 2018

(C) Springer-Verlag GmbH Austria, part of Springer Nature 2018

Dear Editor,

Concerning our contribution "Evaluation of cerebral aneurysm wall thickness in experimental aneurysms: comparison of 3T-MR imaging with direct microscopic measurements" [1], we would like to add the following clarifications. We reanalyzed our initial data after challenging questions of a MR-microscopy specialist and would like to clear the following points: concerning our MR measurements, for both coils, the FOV was $120 \mathrm{~mm} \times$ $120 \mathrm{~mm}$ and the slab thickness was $21 \mathrm{~mm}$, recording 14 slices with $256 \times 256$ pixels. This leads to a spatial resolution of $0.47 \times 0.47 \times 1.5 \mathrm{~mm}^{3}$. Contrary to our statement of not using interpolated images, we retrospectively found out that the software we used for the measurements automatically interpolated the images when zooming in. Therefore, our measurements of $0.05 \mathrm{~mm}$ resolution are based on image interpolations and not direct MR resolution. This is a source of bias, thus a methodological shortcoming and a limitation to our findings.

Camillo Sherif

camillo.sherif@cerebrovascular.at

Cerebrovascular Research Group, Vienna, Austria

2 Department of Neurosurgery, Hospital Rudolfstiftung, Vienna, Austria

3 Medical Faculty, Sigmund Freud Private University, Vienna, Austria

4 High Field MR Center of Excellence, Medical University of Vienna, Vienna, Austria

5 Division of Endocrinology and Metabolism, Department of Medicine III, Medical University of Vienna, Vienna, Austria
We did not intend to claim for a method to boost resolution of 3T MRI. We hereby underline once again explicitly the necessity of research in the field of resolution improvement. To exclude any source of measurement bias from the MRphysical point of view, it is necessary to reach a two times higher resolution than the targeted structure, i.e., when aiming to image $0.4 \mathrm{~mm}$ as proposed cutoff for AWT with high rupture risk, we would need $0.2 \mathrm{~mm}$ resolution for clinical measurements without any bias. Finally, we would like to underline once again our conclusion that further quantitative and comparative experimental and human studies providing this high resolution are warranted.

\section{Reference}

1. Sherif C, Kleinpeter G, Mach G, Loyoddin M, Haider T, Plasenzotti R, Bergmeister H, Di Ieva A, Gibson D, Krssak M (2014) Evaluation of cerebral aneurysm wall thickness imaging with direct microscopic measurements. Acta Neurochir 156:27-34 